\section{POSTER 3-4}

\section{Intra-City Regional Demographics of Major Trauma}

*Stan Feero, MD,1,2 Jerris R. Hedges, $M D, M S,{ }^{2}$

Erik Simmons, ${ }^{3}$ Lisa Irwin, RN, MPA ${ }^{3}$

1. St. Peter Hospital, Emergency Department, Olympia, Washington

2. Oregon Health Sciences University, Department of Emergency Medicine, Portland, Oregon

3. Oregon Department of Human Resources, Health Division, Emergency Medical Services and Trauma System

Objective: To report intra-city, regional geographic and demographic factors affecting risk of major trauma for intentional versus nonintentional trauma.

Design: One-year retrospective association of trauma registry and census tract databases.

Setting: Urban trauma system with patient entry by emergency medical services (EMS) personnel.

Participants: Major trauma cases grouped by presumed intent to injure.

Measurements: Age- and sex-adjusted trauma rates for seven geographic intra-city regions (comprised of 144 census tracts) were associated with population density, median household income, and race data. Rates and risk factors for intentional versus non-intentional trauma were compared.

Results: There were 257 intentional and 575 non-intentional major trauma system cases. Both intentional and non-intentional injury populations were predominately male $(R R=7.04$ and 2.74 , respectively). Intentional trauma was largely a nonwhite phenomenon $(R R=4.08)$ affecting the 15-24 year and 25-34 year age groups (RR = 20.3 and 15.3 respectively). Regional differences in occurrence rates were most pronounced for intentional trauma; $52 \%$ of all intentional trauma cases occurred within a small area of 14 census tracts and was associated with nonwhite race and low median household income for site of injury.

Conclusion: Different demographic features affect intentional versus nonintentional major trauma in Portland, Oregon. These features can be used to guide EMS planning and injury prevention measures.
POSTER 4-42

\section{Application of the Trauma Triage Rule to Blunt Trauma Patients}

\author{
*Andrew Zechnich, MA,1,2 Jon Jui, MD, ${ }^{1}$ Jerris \\ Hedges, MD, ${ }^{1}$ Richard Mullins, MD, ${ }^{3}$ Kent \\ Spackman, $M D, P h D,{ }^{2}$ Phil Feliciano, $M^{3}$ \\ 1. Oregon Health Sciences University, Department of \\ Emergency Medicine \\ 2. Oregon Health Sciences University, Biomedical \\ Information Communications Center \\ 3. Oregon Health Sciences University, Department of Surgery
}

Objective: Baxt recently proposed the Trauma Triage Rule (TTR), reporting 92\% sensitivity and specificity in identifying trauma patients requiring care at level-I centers. Using slightly different methodology and definition of "major trauma," Emerman subsequently reported 94\% sensitivity for the TTR. This investigation measures the accuracy of the TTR in an independent data set.

Methods: Retrospective application of the TTR to trauma registry data. Accuracy in identifying major trauma victims was measured using the resource-based definitions used by Baxt and Emerman. Participants included 626 adult, blunt trauma patients at a level-I trauma center serving a metropolitan area of more than 1-million people.

Results: Of 524 patients with sufficient data, 95 (18\%) and $63(12 \%)$ patients met the criteria for "major trauma" used by Baxt and Emerman, respectively. Using Baxt's definition of major trauma, the TTR revealed a sensitivity of $74 \%$ ( $95 \%$ CI: $0.65-0.83$ ), and a specificity of $84 \%$ ( $95 \%$ CI: $0.81-0.88$ ). There were 25 significant false negative results, including 12 patients requiring urgent laparotomy and four patients requiring emergency airway procedures. Using Emerman's definition of major trauma, sensitivity improved modestly ( $76 \%$ sensitivity (95\% CI: $0.65-0.87)$ and $80 \%$ specificity (95\% CI: $0.77-0.84)$.

Conclusions: In this population, Baxt's TTR failed to identify a significant number of severely injured blunt trauma patients. Slight alterations in the definition of "major trauma" can significantly affect the performance characteristics of triage instruments. 